Asheshov, E. H. \& Rrppon, J. E. (1959). J. gen. Microbiol. 20, 634-643

\title{
Changes in Typing Pattern of Phage-Type 80 Staphylococci
}

\author{
By ELIZABETH H. ASHESHOV AND JOAN E. RIPPON* \\ Staphylococcus Reference Laboratory, Public Health Laboratory Service, \\ Colindale, London, N.W. 9
}

SUMMARY : Three temperate phages isolated from lysogenic staphylococci of phage type 52/52 A/80 were used to lysogenize strains of phage-type 80. Two of these phages belonged to serological group $A$ and one to serological group F. Lysogenization of a strain of phage-type 80 with one of the phages $\left(287^{\prime}\right)$ resulted in a change in the typing pattern from $80 / 81$ to $52 / 52 \mathrm{~A} / 80$. There was, therefore, a gain in sensitivity to phage 52 and $52 \mathrm{~A}$ and a loss in sensitivity to phage 81. Lysogenization of type 80 strains with the second phage (581') caused a loss in sensitivity to phage 81 , while lysogenization with phage $\mathbf{7 2 8 7}^{\prime}$ caused a gain in sensitivity to phage 52 and $52 \mathrm{~A}$.

Loss in sensitivity to phage 81 after lysogenization with phage $287^{\prime}$ seems most probably due to specific prophage immunity since phages $287^{\prime}$ and 81 belong to the same serological group (A) and may be closely related. Loss in sensitivity to phage 81 after lysogenization with phage $581^{\prime}$ does not appear to be a case of specific prophage immunity since the two phages are serologically distinct and the phenomenon resembles examples of prophage interference observed in other organisms.

Rountree \& Freeman (1955) described a number of hospital epidemics in Australia caused by a strain of staphylococcus which was almost completely resistant to the routine typing phages then in use. However, the two group-I phages 52 and 52A produced a few plaques when applied undiluted to a lawn of the organisms, and phage $52 \mathrm{~A}$ was 'adapted' to this new type of staphylococcus. The adapted phage is now known as phage 80 and strains lysed specifically by this phage as phage-type 80 . A second phage known as 81 was isolated independently in Canada (Bynoe, Elder \& Comtois, 1956); it is capable of lysing the majority of phage-type 80 strains. Such strains are therefore more accurately described as phage-type 80/81. Since, however, phage 81 is not included in the set of 21 basic phages used in Great Britain, the strains are usually reported simply as phage-type 80 . The 81 reaction will be referred to in this paper only when relevant.

Since the original description by Rountree \& Freeman many epidemics caused by staphylococci of phage-type 80 have been described in different countries. During the past two or three years it has been noticed in this laboratory that, in some prolonged epidemics caused by phage-type 80 , strains giving the typing pattern 52/52 A/80 were also isolated, particularly during the later part of the epidemic. The 'classical' phage-type 80 coccus is lysed only by phage 80 at the routine test dilution (RTD), using the basic set of

* Present address: National Collection of Type Cultures, Public Health Laboratory Service, Colindale, London, N.W. 9. 
phages. Strains typing as 52/52 A/80 therefore differ from phage-type 80 strains by two reactions and the usual practice in interpreting the results of typing patterns is to consider that strains differing from one another by two or more 'strong' reactions (at the RTD dilution) have a different origin. The frequency with which phage-type 52/52A/80 staphylococci occurred in conjunction with phage-type 80 strains, however, suggested that a close relationship existed between strains giving these two different typing patterns. Investigations were undertaken to see whether such a relationship did exist. It was decided first to see whether lysogenicity was, in any way, responsible for the two different typing patterns. It has been shown by a number of workers (Smith, 1948 $a, b$; Lowbury \& Hood, 1953; Rountree, 1956) that typing patterns of strains of staphylococci are often influenced by the carriage of a particular prophage. It has also been demonstrated in the phage-typing of Salmonella typhi that the presence of a 'type-determining' phage has a direct influence on the typing pattern of a strain (Anderson \& Felix, 1953).

\section{RESULTS}

\section{Tests for lysogenicity}

Staphylococcal strains of phage-types 80 and 52/52 A/80 were examined for lysogenicity; 12 strains of phage-type 80 were examined and 39 strains of phage-type 52/52 A/80. Cultures were grown in nutrient broth for $4-5 \mathrm{hr}$, centrifuged at $3000 \mathrm{rev} . / \mathrm{min}$. for $10 \mathrm{~min}$. and the supernatant fluids spotted on staphylococcal strains of the opposite phage-type. None of the type-80 strains was lysogenic under these conditions; 33 out of the 39 strains of 52/52 A/80 were lysogenic and carried phages capable of lysing and lysogenizing phage-type 80 strains.

\section{Effect of lysogenization on typing patterns}

Stocks of the phage carried by several strains of phage-type 52/52 A/80 staphylococci were prepared by growing the lysogenic cultures in broth for 4-5 hr., centrifuging down the cocci, and filtering the supernatant fluid through sintered-glass filters (APD 0.2 $\mu$ ) or membrane filters (APD 0.25 $\mu$ ). The filtrates were tested for sterility and were then used to lysogenize a number of typical phage-type 80 strains. The filtrate was spotted undiluted on to a lawn of the staphylococci on nutrient agar. Secondary growth in the areas of lysis was visible on the following day and this was picked and streaked on nutrient agar plates to give isolated colonies. Several well-isolated colonies were picked into broth, grown for 4-5 hr. and tested for lysogenicity. They were considered to be lysogenic when (1) the supernatant fluid of a centrifuged sample was shown to contain the particular phage used to lysogenize them and (2) the culture had changed from sensitivity to resistance to this phage (i.e. had become immune). When it was established that the cultures were lysogenic, their typing pattern was tested by using the 21 basic phages in the routine typing set and the 21 extra phages used in this laboratory to type strains which are non-typable with the basic set. 
It was possible to distinguish three distinctly different phages carried by the various strains of phage-type 52/52 A/80. These were: phage $287^{\prime}$ isolated from strain 287; phage 581' isolated from strain 581; phage $7287^{\prime}$ isolated from strain 7287. There are probably others, but these three phages were examined in some detail; their characteristics are summarized in Table 1 which shows only the reactions with four of the typing phages since the strains were resistant to all the other typing phages. These four phages were three of the group I typing-phages (52, 52 A and 80) and phage 81. All of the phage-type 80 staphylococci used in these experiments were lysed equally well by phage 81 at the routine testing dilution and were therefore of phagetype $80 / 81$ before lysogenization. In general our results agree with those reported by Rountree: our phage 581' resembles her phage 863 and our phage 7287' resembles her serological group A phages. Lysogenization with each of the three phages resulted in a change in the typing pattern of the strain lysogenized which took the form of a gain in sensitivity or a loss in sensitivity or a concurrent gain in sensitivity to two phages and a loss to a third. In general the typing pattern resulting from lysogenization corresponded with that of the strain from which the phage was derived.

\section{Table 1. Characteristics of three temperate phages derived from lysogenic strains of phage-type 52/52 A/80}

The typing patterns recorded below were those using the phages at the routine testing dilution. Degrees of reaction recorded as follows: $++=\mathbf{5 0}$ or more plaques; $+=20-50$ plaques; $\pm=$ less than 20 plaques.

\begin{tabular}{|c|c|c|c|c|c|c|c|c|c|c|c|}
\hline \multirow[b]{2}{*}{ Phage } & \multirow{2}{*}{$\begin{array}{l}\text { Sero- } \\
\text { logical } \\
\text { group }\end{array}$} & \multicolumn{4}{|c|}{$\begin{array}{c}\text { Typing pattern of strain } \\
\text { from which phage was } \\
\text { derived }\end{array}$} & \multicolumn{4}{|c|}{$\begin{array}{l}\text { Typing pattern of } \\
80 / 81 \text { strains after } \\
\text { lysogenization }\end{array}$} & \multicolumn{2}{|c|}{$\begin{array}{l}\text { Changes in phage- } \\
\text { sensitivity } \\
\text { following } \\
\text { lysogenization }\end{array}$} \\
\hline & & 52 & $52 \mathrm{~A}$ & 8 & 81 & 52 & $52 \mathrm{~A}$ & 80 & 81 & Gains & Losses \\
\hline $287^{\prime}$ & $\mathbf{A}$ & + & \pm & + & - & + & \pm & ++ & - & $52,52 \mathrm{~A}$ & 81 \\
\hline $581^{\prime}$ & $\mathbf{F}$ & + & $\bar{t}$ & ++ & - & $-*$ & -* & ++ & - & $-*$ & 81 \\
\hline $\mathbf{7 2 8 7 ^ { \prime }}$ & $\mathbf{A}$ & $+t$ & ++ & $+t$ & ++ & ++ & ++ & ++ & ++ & $52, \mathbf{5 2 A}$ & - \\
\hline
\end{tabular}

* The majority of colonies of phage-type 80/81 strains lysogenized with phage 581' were sensitive only to phage 80 . However, occasional colonies showed sensitivity to phages 52 and $52 \mathrm{~A}$ as well.

The three phages were distinguished most easily on the basis of the resistance patterns they conferred against one another when a single strain of phagetype 80 was lysogenized separately with each of the three phages (see Table 2 ). The convention used in designating these indicator strains was to put the number of the carried phage in parentheses after its strain. Thus, strain $6346\left(287^{\prime}\right)$ refers to staphylococcal strain 6346 lysogenized with phage $287^{\prime}$. This set of indicator strains was used to characterize the phages carried by other lysogenic strains of phage-type $52 / 52 \mathrm{~A} / 80$. Of the 33 lysogenic staphylococcal strains tested 15 carried a phage corresponding to phage $287^{\prime}, 2$ carried a phage like 581', and 6 a phage like 7287'. Ten strains carried a phage or phages able to lyse all 4 indicator strains. These differed from any of the three main phages but, as they were not further investigated, it is not known 
whether they represented 'converting' phages, i.e. phages capable of changing the typing pattern. In addition, 6 strains of phage-type 80 which were resistant to phage 81 were examined for lysogenicity. All 6 were lysogenic and carried a phage whose lytic spectrum corresponded to that of phage 581'.

The results suggest that the change in typing pattern occurred as the direct result of lysogenization. The loss in sensitivity to phage 81 after lysogenization with phage $287^{\prime}$ may be due to prophage immunity, since both phage $287^{\prime}$ and phage 81 belong to the same serological group (A) and may be closely related. Moreover, strains of phage-type 80 lysogenized with phage $581^{\prime}$ lost sensitivity to both phage 81 and to phage $287^{\prime}$ (Tables 1 and 2 ).

Table 2. Lytic action of the three phages on a set of indicator strains obtained by lysogenizing a single strain, 6346, of phage-type 80 , with each of the three phages separately

$\begin{aligned} & \text { Indicator } \\ & \text { strains }\end{aligned}$
6346
6346 (287')
$6346\left(581^{\prime}\right)$
$6346\left(7287^{\prime}\right)$

\begin{tabular}{|c|c|c|}
\hline \multicolumn{3}{|c|}{ Phages } \\
\hline $\mathbf{2 8 7 ^ { \prime }}$ & $581^{\prime}$ & $7287^{\prime}$ \\
\hline+ & + & + \\
\hline- & + & + \\
\hline- & - & + \\
\hline+ & + & - \\
\hline
\end{tabular}

Loss of sensitivity to phage 81 after lysogenization with phage $581^{\prime}$ may well be due to prophage interference; it does not appear to be due to specific prophage immunity since the two phages are serologically distinct. A similar phenomenon occurs in Salmonella typhi, where lysogenization with a 'typedetermining' $O$ phage renders the strain resistant to the unrelated $V i$ typing phage (Anderson \& Felix, 1953). It has also been described for the three temperate phages, $\lambda, \mathrm{P} 1$ and P2 (Lederberg, 1957; Bertani, 1958).

Gain in sensitivity to phages 52 and $52 \mathrm{~A}$ after lysogenization with phages $\mathbf{2 8 7}$ ' and $\mathbf{7 2 8 7 ^ { \prime }}$ (and occasionally with phage $\mathbf{5 8 1}$ ) is more difficult to explain. However, it should be noted that staphylococci of phage-type 80 are not completely resistant to phages 52 and $52 \mathrm{~A}$; they are capable of adsorbing the phage particles at an appreciable rate. Adsorption of a single phage particle appears to ' $k$ ill' a coccus in that this is lost as a colony-former. It would appear therefore that phage 52 and phage $52 \mathrm{~A}$ are able to penetrate into cells of phage-type 80 but that their synthesis is blocked at some later stage. It is also worth noting that the three group I phages, 52, 52 A and 80 are phenotypic modifications of a single phage-type and the difference between strains able to synthesize one or all three modifications may be very slight (unpublished observations).

There appeared to be four possible explanations of the gain in sensitivity to phages 52 and $52 \mathrm{~A}$ after lysogenization. These are: (1) lysogenic conversion; (2) selection of pre-existent mutants; (3) transduction; (4) prophage substitution. Several experiments to test the validity of these various possibilities were carried out. All of these experiments were done with staphylococci of strain 6346, phage-type 80 and phage $7287^{\prime}$. 
Lysogenic conversion would appear to be the simplest explanation of the gain in sensitivity to phages 52 and $52 \mathrm{~A}$ in lysogenized phage-type 80 cells. It assumes that the presence of the prophage is necessary to overcome the block in the synthesis of the two phages noted above, in the same way that the presence of the prophage is necessary to convert non-toxigenic strains of Corynebacterium diphtheriae to toxin-producing strains (Freeman, 1951). The simplest way to test this explanation appeared to be to 'cure' the lysogenic strain and see whether it would revert to phage-type 80. The method used to isolate non-lysogenic mutants in the lysogenic culture was that described by Lederberg \& Lederberg (1953). Two different lysogenic strains were included in these experiments. Strain 7287 of phage-type 52/52 A/80 from which phage $7287^{\prime}$ was isolated; strain $6346\left(7287^{\prime}\right)$, i.e. strain 6346 of phage-type 80 , lysogenized with phage $\mathbf{7 2 8 7}^{\prime}$ and thus converted to phage-type 52/52 A/80. Broth cultures of both strains were used to flood nutrient agar plates containing $2 \times 10^{-2} \mathrm{M}$-sodium citrate. The plates were u.v. irradiated sufficiently to eliminate all but 100-200 colonies/plate (about 1 in $10^{6}$ survival). These colonies were then replicated on plates flooded with a suitable indicator strain (6346). This made it possible to distinguish between lysogenic and nonlysogenic colonies, because the former were always surrounded by a narrow zone of phage lysis. Non-lysogenic mutants were isolated from each of the two staphylococcal strains; both proved to be of phage-type 52/52 A/80. The continued presence of the prophage $\mathbf{7 2 8 7}^{\prime}$ was therefore not necessary to maintain the change of phage-type 80 strains to phage-type 52/52 A/80, and the observed change in typing pattern could not be due to simple lysogenic conversion.

Selection of pre-existing mutants. This explanation was first proposed by Rountree (personal communication, and 1959). It supposes that the converting phage acts as a selector of pre-existent mutants. Rountree found that old cultures of phage-type 80/81 staphylococcal strains contained mutants of phage-type $52 / 52 \mathrm{~A} / 80 / 81$ which she was able to isolate by a replica plating technique. The mutants were present in the proportion of $c .1 / 200$ cocci in $24 \mathrm{hr}$. broth cultures. She suggested that the action of the 'converting' phages was a direct selective one-i.e. the phages lysed the parent $80 / 81$ cocci and lysogenized the mutant $52 / 52 \mathrm{~A} / 80 / 81$ cocci. The mutants therefore would be the only cocci to survive exposure to the phage and would survive by virtue of their being lysogenized. We attempted to isolate these mutants from strain 6346 by using the replica plating method, but failed. A total of 5774 colonies was examined in five different experiments, the highest number in any one experiment being 1465 . We used 4-5 hr. broth cultures of strain 6346, and it is quite possible that the mutants were not present at that time or were not present in sufficient concentration to be detected by the screening method. It seemed, however, that we had an example of the mutant in the culture of strain 6346 which was first lysogenized with phage $7287^{\prime}$ and subsequently de-lysogenized (see above); this strain is referred to as $6346 \mathrm{DL}$. Experiments were carried out to compare the frequency of lysogenization of strain 6346 with that of strain $6346 \mathrm{DL}$, using phage $7287^{\prime}$. If the selection 
explanation were correct the frequency of lysogenization of strain $6346 \mathrm{DL}$ should be much higher than that of strain 6346 .

The experiments were carried out as follows. The two strains were grown in broth for $4 \mathrm{hr}$. to c. $5 \times 10^{7}$ colony-formers $/ \mathrm{ml}$. Each culture was then divided into two parts. Phage $\mathbf{7 2 8 7}^{\prime}$ was added to one part to give $1 \times 10^{9}$ phage particles/ml., so that there were about 20 phage particles/colonyformer; this is referred to as the adsorption mixture. The other half of the culture served as a control of the initial concentration of bacteria (bacterial control) and was examined for the presence of mutants of phage-type 52/52 A/ 80. The adsorption mixtures were shaken at $37^{\circ}$ for $10 \mathrm{~min}$. to allow phage adsorption and were then diluted $1 / 10$ with broth containing sufficient antiserum to inactivate $99.5 \%$ of unadsorbed phage in 5 min. Five minutes were allowed for serum inactivation and the tubes were further diluted to contain 100-200 colony formers $/ 0.1 \mathrm{ml}$; this volume was then spread on each of several plates. The same dilution was made from the bacterial control tubes and similarly plated. After overnight incubation counts were made of the colonies produced from the bacterial control tubes and from the adsorption mixtures. The survivors from the adsorption mixtures were replicated on plates flooded with staphylococcal strain 6346 , in order to detect lysogenic colonies amongst the survivors. Colonies corresponding to those which appeared to be lysogenic were then picked from the master plates and tested for lysogenicity and sensitivity to phages 52, 52 A and 80. Colonies on the plates from the bacterial control tubes were replicated on plates flooded with phage 52, in order to detect any mutants of phage-type 52/52 A/80.

In each of three experiments with strain 6346 we were unable to detect any mutants of phage-type 52/52 A/80 in the bacterial control. All of the colonies from the bacterial control of strain $6346 \mathrm{DL}$ were sensitive to phage 52 when tested by the replica-plate method. This was the expected result and was included as a control on the method of testing for phage 52-sensitive colonies. The results of the platings from the two adsorption mixtures (6346 and $6346 \mathrm{DL}$ ) are given in Table 3. In analysing the results we have listed four

\section{Table 3. Frequency of lysogenization of strains 6346 and $6346 \mathrm{DL}$ by phage $7287^{\prime}$}

Type of response following $10 \mathrm{~min}$. exposure to phage

Staphylococcal
strain
6346
$6346 \mathrm{DL}$

\begin{tabular}{cccc}
\hline $\begin{array}{c}\text { Lytic } \\
(a)\end{array}$ & $\begin{array}{c}\text { Lysogenic } \\
(b)\end{array}$ & $\begin{array}{c}\text { Mixed } \\
(c)\end{array}$ & $\begin{array}{c}\text { Non-lysogenic } \\
(d)\end{array}$ \\
$\mathbf{8 7} \%$ & $\mathbf{1 . 5 7} \%$ & $\mathbf{8 . 8} \%$ & $\mathbf{2 . 6 1} \%$ \\
$\mathbf{8 8 . 5} \%$ & $\mathbf{2 . 0 5} \%$ & $\mathbf{8 . 2} \%$ & $\mathbf{1 . 3 1} \%$
\end{tabular}

Frequency of lysogenization (b) $\overline{(a)+(b)+(c)}$ 0.016 0.020

possible responses : lytic, lysogenic, mixed, non-lysogenic. The \% cocci which gave a lytic response was calculated by subtracting the number of survivors from the initial concentrations of cocci, represented by the bacterial control. Amongst the survivors, colonies were considered to be lysogenic when they

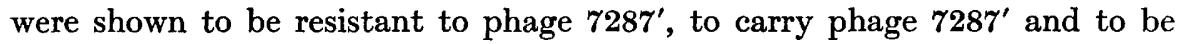
stable on subculture. All the lysogenic colonies were found to be of phage- 
type 52/52 A/80. A large proportion of the survivors were classified as ' mixed '; these were unstable and tended to undergo spontaneous lysis on subculture. As a rule they were partially resistant to phage $7287^{\prime}$; they were shown to be carrying phage $\mathbf{7 2 8 7}^{\prime}$ but appeared to consist of a mixture of phage-sensitive and lysogenic phage-resistant cocci. They probably arose originally from clumps of cocci of which only a proportion became lysogenized during the $10 \mathrm{~min}$. adsorption period, the rest remaining non-lysogenic and phage-sensitive. Non-lysogenic colonies were tested in a few instances and proved to be fully sensitive to phage $\mathbf{7 2 8 7 ^ { \prime }}$; these colonies presumably arose from cocci which had not become infected during the adsorption period. The results of these experiments did not support the selection explanation, for two reasons.

(a) We were unable to detect the mutants of phage-type 52/52 A/80 in the bacterial control suspension of strain 6346 , although $1.57 \%$ of the cocci gave a stable lysogenic response when exposed to phage $7287^{\prime}$ and were phagetype 52/52 A/80. However, in interpreting the results one fact needs to be considered. Only a small proportion of the colonies in the experiments arose from single cocci. Smears made from the suspensions of cocci immediately before beginning the experiment and examined microscopically showed that the average unit consisted of between 2.5 and 3.0 cocci. This undoubtedly explains why so many of the survivors from the adsorption mixtures gave mixed responses. It is probable that this fact biased the results against support of the selection explanation. If a mutant is present as one coccus in a clump of 4 cocci, the colony which develops from this clump will contain only $\mathbf{2 5} \%$ of cocci susceptible to phage $\mathbf{5 2}$ (assuming identical growth rates for the mutant and the wild-type cocci). Whether such a colony would be recognizable as sensitive to phage $\mathbf{5 2}$ by the replica-plating method seems doubtful. If the same clump of cocci were exposed to phage $\mathbf{7 2 8 7}^{\prime}$ and only the mutant became lysogenized, the colony that formed would be classified as giving a lysogenic or a mixed response, depending on how soon the other, phagesensitive, non-lysogenic cocci were eliminated by lysis with phage 7287'.

(b) Comparison of the frequency of lysogenization of strain 6346 and 6346DL failed to show any significant differences although, on the basis of our second explanation (selection of pre-existing mutants) strain 6346DL should have shown a much higher frequency than strain 6346. Our general conclusion is therefore that this explanation is not tenable.

Transduction. The assumption that the change in typing pattern was due to transduction in a manner similar to that described for other organisms was eliminated for two reasons: (i) transduction would need to be extremely efficient, since all of the lysogenized colonies tested were found to have the new typing pattern; (ii) the character of the strain on which the 'converting' phage was last propagated did not affect the change; phage $7287^{\prime}$ propagated on strain 6346 (phage-type 80 ) gave the same results as phage $7287^{\prime}$ derived by spontaneous lysis of strain 7287 (phage-type 52/52 A/80).

Prophage substitution. The fourth possibility was that cocci of phage-type 80 were originally lysogenic and that lysogenization with a 'converting' phage caused prophage substitution with the loss of the original prophage. Assuming 
that the presence of the original prophage inhibited synthesis of phage $\mathbf{5 2}$ and $52 \mathrm{~A}$, substitution with another phage might have the effect of overcoming this inhibition and of allowing synthesis to occur. In all our experiments the different strains of phage-type 80 were tested for lysogenicity by using strains of the opposite phage-type (52/52 A/80) as indicator strains; in no instance was any phage detected. This failure may have been due to the unsuitability of the indicator strains. Further tests were made by using a single strain of phage-type 80 (strain 6346) as source of the hypothetical phage and using as indicators strains of 6364 lysogenized separately with each of the three 'converting' phages. If prophage substitution had occurred these lysogenized strains should have been sensitive to the phage that was lost and have served as suitable indicators for such a phage. No phage was detected. Filtrates of strain 6346 were then tested on two strains of staphylococci known to be sensitive to the majority of staphylococcus phages; but again no phage was detected. The experiments described by Rountree (1959) indicated that prophage substitution was not responsible for the change in typing patterns. It is, of course, possible that strains of phage-type 80 are really lysogenic but produce only 'defective' phage which cannot mature to produce lysis. There is no evidence for this at the present.

\section{DISCUSSION}

The present results show that the typing pattern of phage-type 80 strains of staphylococci can be changed as the result of lysogenization in vitro with specific temperate phages derived from strains of phage type 52/52 A/80. With three different phages, three different patterns were obtained. With two of the three phages lysogenization caused a loss in sensitivity to phage 81, and with two of the phages there was consistently a gain in sensitivity to phages 52 and 52A. No experiments were carried out to explain the loss in sensitivity to phage 81 , but we consider that in one case the effect was probably due to specific prophage immunity and in the other case to prophage interference.

Gain in sensitivity to phage following lysogenization is a more unusual observation, although not unique. Uetake, Luria \& Burrous (1958) reported that strains of Salmonella anatum became sensitive to phage $\mathrm{E}^{34}$, to which they had hitherto been resistant, as the result of lysogenization with phage $\mathbf{E}^{15}$; they showed that this gain was due to production of a new somatic antigen which was the receptor for phage $E^{34}$. Brandis \& Storch (1957) reported a gain in sensitivity to phage $\mathbf{3 a}$ and $\mathbf{3 a I}$ in strains of $S$. paratyphi $\mathrm{B}$ after lysogenization with a temperate phage (St). The phenomenon was due to recombination between phage $S t$ and the typing phage $3 a$ and $3 a I$ respectively.

The gain in sensitivity to phage 52 and $52 \mathrm{~A}$ reported here is not due to a change in the phage receptor sites on the cell wall of the staphylococei used, since phage-type 80 cocci absorbed both phages at a measurable rate. The block in the synthesis of the two phages appears to occur at a later stage, after adsorption and probably after penetration, because there is evidence that adsorption of either phage killed phage-type 80 cocci. Four explanations were 
put forward to explain the phenomenon (transduction, lysogenic conversion, selection of pre-existent mutants, prophage substitution) but the results failed to support any of these. Transduction appeared unlikely because of the high efficiency of the change, and was excluded when it was found that the ability of the phage to produce the change was independent of the character of the last strain used to propagate the phage. Selection of pre-existent mutants appeared probable, since Rountree had shown that mutants of phage-type 52/52 A/80 existed in old cultures of phage-type 80 strains. However, studies of frequency of lysogenization in other systems showed that, at least, in these other systems, the frequency of lysogenization depended on the physiological state of the host organisms and could be altered by altering the external conditions (Lieb, 1953; Bertani \& Nice, 1954). The few experiments we made with one staphylococcal strain of phage-type 80 and one of the three 'converting' phages suggested that the frequency of lysogenization was too high to be accounted for by selection of pre-existent mutants.

We were unable to prove or disprove the occurrence of prophage substitution, since no active phage could be demonstrated in filtrates of cultures of phage-type 80 cocci. Our failure may have been due to lack of a suitable indicator strain or because phage-type 80 cocci produced only 'defective' phage. That phage-type 80 cocci may contain an active phage is shown by an observation of Rountree (1959) who found that one staphylococcal strain of phage-type 80 (the propagating strain for phage 80 ) carried a phage of serological group $\mathbf{F}$ which was released both by the original strain and by the strain after lysogenization with one of the 'converting' phages. All that we can say is that there is no evidence so far to support the explanation of prophage substitution.

Lysogenic conversion, as generally understood, cannot explain the results, because the change in typing pattern remained after the loss of the 'converting' phage. In other cases of lysogenic conversion, loss of prophage has resulted in a reversion to the pre-lysogenic character (e.g. Groman, 1955). However, in the absence of any other explanation and because of the available experimental evidence, we suggest that the change in typing pattern occurs as the direct result of lysogenization, but that once this change has occurred it remains and is independent of the continuing presence of the prophage.

The work reported here originated in the observation that staphylococcal strains of typing pattern 52/52 A/80 seemed to appear with undue frequency in epidemics due primarily to type-80 strains. We have since seen six cases in which staphylococci with both typing patterns were isolated from the same person. Bearing in mind the great number of phage patterns among staphylococci, this is strong evidence that the change from the 80 to the 52/52 A/80 pattern, or the reverse, can occur in nature. In fact, the epidemiological evidence suggests that the change is usually from 80 to $52 / 52 \mathrm{~A} / 80$. But further investigation is needed on this point because strains of type 80 are undoubtedly much more often the cause of epidemics of hospital infection than are strains with the pattern $52 / 52 \mathrm{~A} / 80$. 


\section{REFERENCES}

Anderson, E. S. \& Felix, A. (1953). The Vi-type determining phages carried by Salmonella typhi. J. gen. Microbiol. 9, 65.

Bertani, G. (1958). Lysogeny. Advanc. Virus Res. 5, 151.

Bertani, G. \& Nice, S. J. (1954). Studies on lysogenesis. II. The effect of temperature on the lysogenization of Shigella dysenteriae with phage P1. J. Bact. 67, 202.

Brandis, H. \& Storch, I. (1957). Uber eine Kreuzung von Paratyphus B-Bakteriophagen. Z. Hyg. InfektKr. 144, 164.

Bynoe, E. T., Elder, R. H. \& Comtois, R. D. (1956). Phage-typing and antibiotic-resistance of staphylococci isolated in a general hospital. Canad. J. Microbiol. 14, 167.

Freeman, V. J. (1951). Studies on the virulence of bacteriophage-infected strains of Corynebacterium diphtheriae. J. Bact. 61, 675 .

Groman, N. (1955). Evidence for the active role of bacteriophage in the conversion of non-toxigenic Corynebacterium diphtheriae to toxin production. J. Bact. 69, 9.

Lederberg, E. M. \& Lederberg, J. (1953). Genetic studies of lysogenicity in Escherichia coli. Genetics, 38, 51.

LEDERBERG, S. (1957). Suppression of multiplication of heterologous bacteriophages in lysogenic bacteria. Virology, 3, 496.

LIEB, M. (1953). The establishment of lysogenicity in Escherichia coli. J. Bact. 65, 642.

LowBury, E. J. L. \& Hood, A. M. (1953). The acquired resistance of Staphylococcus aureus to bacteriophage. J. gen. Microbiol. 9, 524.

Rountree, P. M. \& Freeman, B. M. (1955). Infections caused by a particular type of Staphylococcus aureus. Med. J. Aust. ii, 157.

Rountree, P. M. (1956). Variations in a related series of staphylococcal bacteriophages. J. gen. Microbiol. 15, 266.

Rountree, P. M. (1959). Changes in the phage-typing patterns of staphylococci following lysogenization. J. gen. Microbiol. 20, 620 .

SMrth, H. W. (1948a). Investigations on the typing of staphylococci by means of bacteriophage. I. The origin and nature of lysogenic strains. J. Hyg., Camb. 46, 74 .

Sмттн, H. W. $(1948 b)$. Investigations on the typing of staphylococci by means of bacteriophage. II. The significance of lysogenic strains in staphylococcal type designation. J. Hyg., Camb. 46, 82.

Uetake, H., Luria, S. E. \& Burrous, J. W. (1958). Conversion of somatic antigens in Salmonella by phage infection leading to lysis or lysogeny. Virology, 5, 68.

(Received 1 December 1958) 